East African Medical Journal Vol. 87 No. 4 April 2010

PROVIDER DELAY IN THE DIAGNOSIS AND INITIATION OF DEFINITIVE TREATMENT FOR BREAST CANCER PATIENTS

E.S. Otieno, MBChB, MMed (Surg), PGDRM, Lecturer, Department of Medical Physiology, College of Health Sciences, University of Nairobi, P.O. Box 5510-00100, Nairobi, Kenya, J.N. Micheni, MBChB, MMed (Surg.), Consultant Surgeon and Director, Kenyatta National Hospital, P.O. Box 19961 - 00202, Nairobi, Kenya, S.K. Kimende, MBChB, MMed (Surg.), Lecturer, Department of Surgery, College of Health Sciences, University of Nairobi, P.O. Box 19176-00202, Nairobi, Kenya and K.K. Mutai, BSc, PGDRM, Epidemiologist, P.O. Box 24296-00100, Nairobi, Kenya

Request for reprints to: Dr. E. S. Otieno, Department of Medical Physiology, College of Health Sciences, University of Nairobi, P.O. Box 5510-00100, Nairobi, Kenya

\title{
PROVIDER DELAY IN THE DIAGNOSIS AND INITIATION OF DEFINITIVE TREATMENT FOR BREAST CANCER PATIENTS
}

\author{
E.S. OTIENO, J.N. MICHENI, S.K. KIMENDE and K.K. MUTAI
}

\begin{abstract}
Objective: To determine the extent and nature of provider delay in breast cancer management at Kenyatta National Hospital (KNH).

Design: Retrospective descriptive study.

Setting: Kenyatta National Hospital breast clinic.

Subjects: Records of 500 patients were reviewed over a four year period.

Result: Out of the 500 patient files reviewed a total of $111(22.2 \%)$ were excluded because either whole or part of their diagnostic work-up was done outside KNH $(n=66)$ or they had recurrent disease $(n=45)$, thus leaving 389 eligible for analysis. Mean overall provider delay (time lapse between the patients' first hospital visit date to time definitive anti-cancer treatment was started) was 87.9 days, ( $=160$, range 1 to 1683 days) and a median of 21.5 days. Nine (5.6\%) patients were started on treatment three or more years after they initially presented to the hospital. Mean delay with regard to confirmatory laboratory diagnostic test was 56.2 days ( $n=83$, range 1 to 985 days, standard deviation 146.7) with a median of 17.0 days. Eighty seven $(22.4 \%)$ patients had complete data set to assess for the lapse in time from definitive diagnosis to the time definitive anti-cancer treatment was initiated. This mean delay was 93.5 days with a median of 28.0 days.

Conclusion: The median overall provider delay for patients diagnosed with breast cancer at KNH is reasonable and compares well with that of other institutions. It remains to be determined why a small number of patients take unusually long to be put on definitive anti-cancer treatment.
\end{abstract}

\section{INTRODUCTION}

Undue delay during the diagnostic workup of patients with breast cancer is known to result in more adverse outcomes (1). Two types of delay are well recognised with respect to breast cancer management. The first type, occasioned by the patient considers time lapse between noticing the breast symptom and presenting to the healthcare provider (patient delay). The second being time lapse between presentation to a health care provider or facility and initiation of definitive cancer treatment (provider delay) $(1,2)$. It has been consistently reported that patient delay of more than three months is associated with higher rates of recurrence and worse overall survival (3-5). Literature on provider delay, on the other hand is scarce and indeed its impact on both recurrence and overall survival is not clear (5). The causes of provider delay are complex and may include patient non-compliance, provider misdiagnosis and long waiting periods for diagnostic and therapeutic procedures owing to the limited capacities of healthcare systems as well as absence of clear diagnostic policies. Provider delay is indeed a measure of efficiency with which any given health care system delivers timely services. In addition it is a source of significant psychological stress to the patient (6). Where breast self examination (BSE) is widely and effectively practised, the diagnostic delays inherent in our healthcare systems may nullify the potential benefits that accrue from timely presentation following BSE (7). The median provider delay varies from 14 to 30 days in studies done in Europe and North America $(9,10)$.

Existing literature on provider delay mainly comes from the Western World with very littleindeed written from the developing countries. Yet, it is envisaged that 
provider delay may be worse in these regions, owing to scarcity of resources and manpower constraints. Quality and timely care remains a central goal of any breast unit specialising in breast disease management and any delay during diagnostic workup for cancer should indeed be kept to the minimum. This study undertook toevaluate the extent and nature of provider delay at KNH breast clinic.

\section{MATERIALS AND METHODS}

Study design and setting: Retrospective descriptive study over a four year period from the $1^{\text {st }}$ October 2000 to $31^{\text {st }}$ October 2004 carried out at Kenyatta National Hospital breast clinic. The KNHbreast clinic was set up in the year 2000 as a specialist clinic for the purpose of managing all diseases of the breast. It is held once a week and run by consultant surgeons and surgical residents in training.

Study population: All patients seen at the KNH breast clinic during the study period with cytologically or histologically confirmed breast cancer were included in this study. Patients whose diagnostic work up and / or treatment were wholly or partially carried out outside Kenyatta National Hospital were excluded from the study. Also excluded were patients who presented for the first time at KNH breast clinic with recurrent disease.

Data collection: All case files and medical records of patients seen at the breast clinic during the study period and who met the inclusion criteria were retrieved and relevant clinico-demographic data entered into a structured questionnaire. Data collected included the date when patient was first seen at $\mathrm{KNH}$, date first seen at the breast clinic, date that the confirmatory test was asked for, date this test was actually taken/done, the date this test was reported and the date the first definitive anti cancer treatment was initiated. (Initial anti-cancer treatment was accepted as surgery, radiotherapy, chemotherapy and/ or hormonal therapy either singly or in combination.
Ethical issues: Written permission to do the study was granted by the Kenyatta National Hospital Research and Ethic Committee.

\section{RESULTS}

In this retrospective study over a four year period 500 patients' files were reviewed. Sixty six of these had part or whole of their diagnostic work up done in other hospitals and a further 45 patients presented with recurrent disease and thus were excluded leaving 389 eligible patients for analysis. The mean age was 44 years $(n=389)$, and the range was 17 to 99 years. Females constituted $96.6 \%(n=384)$. The socio-demographic characteristics of these patients are summarised in Table 1. Data on 160 out of the $389(41.1 \%)$ patients was complete for assessment of overall provider delay, defined as time lapse between when patient presented to the hospital and when definitive anti-cancer treatment was initiated. The mean overall provider delay in this study was 87.9 days (range 1 to 1683 days and std. deviation 248.9) with the median of 21.5 days. Nine patients were started on treatment three or more years after they presented to the hospital.

Out of these 160 patients, $67(41.9 \%)$ were started on treatment within 14 days (range 1 to 14 days, mean 4.2 days, median 2.0 days, standard dev. 21.9). Table 2 shows the number out of these 160 that were started on treatment within 30, 60 and 90 days of presenting at Kenyatta National Hospital

Out of the 389 patients $83(21.3 \%)$ had complete data to assess the time it took from the date confirmatory laboratory request was written to the date it was reported. This so called mean laboratory delay was found to be 56.2 days (range 1 to 985.0 days, std. dev. 146.7) with a median of 17.0 days.

Eighty seven (22.4\%) were assessed for timelapse between when definitive diagnosis was made and when definitive anti-cancer treatment was initiated. This mean delay was found to be 93.5 (range 1 to 1637 days, std dev. 228.46) and a median of 28.0 days.

Table 1

Socio-demographic characteristic of study population

$$
\text { Sex }(n=384)
$$

\begin{tabular}{|c|c|c|c|c|}
\hline Parameter & Male $(\%)$ & Female $(\%)$ & Age range (years) & $\begin{array}{c}\text { Mean age (years) } \\
(\mathrm{n}=389)\end{array}$ \\
\hline Statistics & $13 \quad(3.4)$ & $371 \quad$ (96.6) & $17-99$ & 44 \\
\hline
\end{tabular}


Table 2

Overall provider delay within the first three months

\begin{tabular}{lllllll}
\hline \multirow{2}{*}{$\begin{array}{l}\text { Provider } \\
\text { delay (days) }\end{array}$} & \multicolumn{7}{c}{ Statistical parameters } \\
& No. & Cumulative & mean & median & Std. dev. & $\begin{array}{c}\text { Range } \\
\text { (days) }\end{array}$ \\
\hline 14 & 67 & 41.9 & 4.2 & 2.0 & 3.8 & $1-14$ \\
30 & 95 & 59.4 & 9.3 & 6.0 & 8.8 & $1-29$ \\
60 & 122 & 76.2 & 16.4 & 10.0 & 15.8 & $1-54$ \\
90 & 134 & 83.7 & 21.3 & 14.5 & 21.9 & $1-86$ \\
\hline
\end{tabular}

\section{DISCUSSION}

This study retrospectively reviewed 500 files of patients diagnosed with breast cancer over a four year period at KNH breast clinic. Sixty six files were excluded since part or whole of .their diagnostic procedures had been done in other hospitals and a further forty five were excluded because they presented with recurrent disease leaving three hundred and eighty nine files eligible for analysis. One hundred and sixty $(41.1 \%)$ of the eligible group had complete data set for assessment of overall provider delay, defined as time lapse from presenting to the hospital and initiation of definitive anti-cancer treatment. The mean overall provider delay was 87.9 days (range 1 to 1683 days and std. deviation 248.9) with a median of 21.5 days. This means that half the population under study were indeed started on definitive anti-cancer treatment within three weeks of presentation. This compares favorably well with studies donein other parts of the world where median provider delay has been found to vary from 14 to 30 days in studies done in Europe and North America $(9,10)$. In a population based study in German, Volker et al (5) found a median provider delay of 15 days and concluded that the diagnostic work up was within a reasonably short time limit. Nine $(5.6 \%)$ patients in our study had curiously lengthy provider delays ranging from three to over five years and this is largely responsible for the prolonged mean provider delay of three months reported in this study. In our study only $59.4 \%$ of patients had their treatment initiated within 30 days and $16.3 \%$ were started on treatment after three months compared to $73 \%$ and $11 \%$ respectively in the German study.

The extent of provider delay in any institution reflects the efficiency with which timely services are offered and where breast self examination (BSE) is widely and effectively practiced the diagnostic delays inherent in our health care systems may nullify the potentialbenefits that accruefrom timely presentation following BSE (7). Alcoe and Gilbey (8) studied the experience of eight women who attended BSE classes and later developed breast cancer. From this study they concur that there is need to address the health care system's response to women who present early on the basis of findings through BSE.

Determinants of provider delay are varied. Known factors that have been associated with statistically significant provider delay are the patient's level of education, occupation, presentation with breast symptoms other than a lump, family history of breast cancer, employment status and mode of detection of breast disease. Patient non-compliance was found common in the more educated and professional woman, probably related to time constraints. Volker et al (5) found that women in full time employment had a statistically significant longer period for diagnostic work up. This underlies an important perception among these women, that other priorities take precedence over personal health, and calls for targeted education.

One of the physician causes of provider delay continues to be enthusiastic reassurance of the patient that a breast lesion is benign without recourse to biopsy. Also younger age and presentation with a breast symptom other than a lump were strong risk factors for provider delays (9). A low index of suspicion coupled with the opinion of low mammographic reliability for cancer diagnosis at this young age have been recognised as sources of physician delay (11). Although this study set out to explore the extent to which these factors contribute to provider delay, retrospectively sourced data were largely incomplete for meaningful analysis and a prospective study is therefore recommended.

This study looked at the delay occasioned from the time the laboratory request for tissue biopsy is written and the time the report is actually read. This aspect of provider delay was found to have a mean delay time of 56.2 days for the $83(21.3 \%)$ patients whose data were complete for this assessment (range 1 to 985.0 days, standard deviation 146.7) with a median of 17.0 days. This implies that though on average it took two months for the definitive diagnostic report to be available, half of the population analyzed for this factor, encouragingly had a report ready within two weeks of request. Two hundred and twenty seven $(70 \%)$ of the diagnosis was made by fine needle aspiration (FNAC). The process involved in diagnostic 
reporting is that a patient has a request written and is then given a booking for the day that the FNAC is to be done after which the specimen is taken to the pathology laboratory for processing and reporting. It would be of interest to prospectively determine at which of these stages there is undue delay and why.

Also determined was the timelapse from the date the diagnostic report was made to the time definitive anti-cancer treatment was initiated. This provider delay had a mean of 93.5 days (range 1 to 1637 days, standard deviation 228.46) and a median of 28.0 days. The length of time taken here may reflect more of the way patients are booked to be reviewed with result and calls for a review of booking procedures. Also innovative ways may be employed to ensure that patients whose results are already out can be re-called earlier irrespective of their next appointment time. Other factors that may cause this delay include delays in dispatching the results to the clinic, constraints in theatre time leading to re-bookings and indeed patient non-compliance with regard to keeping appointments. It is recommended that prospective studies bedone to specifically elucidate the truenature of provider delay with respect to these factors.

\section{ACKNOWLEDGEMENTS}

To the Director, Kenyatta National Hospital for allowing us to access the hospital records and granting permission to publish this paper. I would like to acknowledge the hard work done by Hellen Cherono and Peter Oyiro who were both medical students at the time.

\section{REFERENCES}

1. Burgess, C.C., Hunters, M.S. and Ramirez, A.J. A qualitative study of delay among women reporting symptoms of breast cancer. Br. J. Gen. Pract. 2001; 51: $967-971$.
2. Granfeldi, E.A., Ramirez, A.J., Hunters, M.S. and Richards, M.A. Women's knowledge and belief regarding breast cancer. Br. J. Cancer. 2002; 1373.

3 Richards, M.A., Smith, P., Ramirez, A.J. et al. The influence on survival of delay in the presentation and treatment of symptomatic breast cancer. Br. J. Cancer. 1999; 79: 858 - 864.

4. Richards, M.A., Westcomber, A.M., Lore, S.B., et al. Influence of delay on survival in patients with breast cancer; A systematic review. Lancet. 1999; 353: 11191126.

5. Volker, A., Til, S., Christa, S. et al. Provider delay among patients with breast cancer in Germany: A population based study. J. Clin. Oncology. 2003; 8: 1440-1446.

6. Brett, J., Austoker, J. and Ong, G. Do women who undergo further investigations for breast screening suffer adverse psychological consequences? Amulticentre follow-up study comparing different breast result groups 5 months after their last breast screening appointment. J. Pub. Health Med. 1998; 20: 396 - 403.

7. Unknown authors: Reducing diagnostic delay in breast cancer; possible therapeutic implications: GIVID -Italy. Cancer. 1997; 15: 581756 -581761.

8. Alcoe, S.Y. and Gilbey, M.J. From attending a class on breast self examination (BSE) to coping with breast cancer: the experience of eight women. Patient Educ. Couns. 1996; 28:133-148.

9. Ramirez, A.J., Westcombe, A.M., Burgess, C.C. et al. Factors predicting delayed presentation of symptomatic breast cancer: A systemiatic review. Lancet. 1999; 353: 1127 - 1131.

10. Caplan, L.S., Helzlsover, K.J., Shapiro, S. et al. System delay in breast cancer in whites and blacks. Am. J. epidemiol. 1995; 142: $804-812$.

11. Olwetti, L., Bergonzini, R., Vanolic, et al. Is mammography useful in the detection of breast cancer in women 35 years of age and younger. Radiol. Med. (Torino). 1998; 95: 161. 